\title{
Gestión del presupuesto por resultados y calidad del gasto en la municipalidad provincial de Satipo, 2020
}

\author{
M.Sc. Ludy Vílchez Casas \\ ludyvilchez@hotmail.com \\ ludyvilchez@gmail.com \\ Orcid:0000-0001-8205-5803
}

Universidad Nacional Federico Villarreal

\section{RESUMEN}

El propósito de la investigación fue “determinar la relación entre la gestión del presupuesto por resultados y la calidad del gasto de la Municipalidad Provincial de Satipo". La población y muestra fue de 28 servidores públicos relacionados con el ciclo de gasto. La investigación obedece al diseño no experimental de enfoque cuantitativo, alcance correlacional de tipo aplicada. Para la confiabilidad del cuestionario tipo Likert se hizo uso del coeficiente de alfa de Cronbach con 0.914. Los resultados fueron que existe una relación directa y significativa entre la gestión de presupuesto por resultados y la calidad del gasto con un valor de Pearson de 0.693. Además, se obtuvo el $61 \%$ de ejecución financiera y el $43.9 \%$ de las inversiones se orientaron a resultados, sin embargo, no sé evalúa el impacto generado por las inversiones y/o actividades. Por lo tanto, se concluye que, si se da una buena dirección en la asignación de los fondos públicos entonces mejora la eficacia de las inversiones, para lo cual se recomienda que el diseño del presupuesto debe enfocarse a programas presupuestales con cierre de brechas.

Palabras clave: gestión del presupuesto; resultados; calidad del gasto 


\title{
Budget management by results and quality of spending in the provincial municipality of Satipo, 2020
}

\begin{abstract}
The purpose of the research was "to determine the relationship between the management of the budget by results and the quality of the expenditure of the Provincial Municipality of Satipo". The population and sample consisted of 28 public servants related to the spending cycle. The research obeys the non-experimental design of a quantitative approach, correlational scope of the applied type. For the reliability of the Likert-type questionnaire, the Cronbach's alpha coefficient was used with 0.914 . The results were that there is a directive and significant relationship between budget management by results and the quality of spending with a Pearson value of 0.693 . In addition, $61 \%$ of financial execution was obtained and $43.9 \%$ of the investments were results-oriented, however, the impact generated by the investments and / or activities is not evaluated. Therefore, it is concluded that, if a good direction is given in the allocation of public funds, then the effectiveness of investments improves, for which it is recommended that the design of the budget should focus on budget programs with closing gaps.
\end{abstract}

Keywords: budget management; results; quality of spending

Artículo recibido: 05 octubre. 2021 Aceptado para publicación: 02 noviembre 2021 Correspondencia: : ludyvilchez@gmail.com Conflictos de Interés: Ninguna que declarar 


\section{INTRODUCCIÓN}

El modelo de Evaluación PRODEV (SEP), ha sido desarrollado con el propósito de evaluar la articulación de los componentes de gestión de los países de América Latina y el Caribe y a su vez como estos vienen implementando la Gestión para Resultados en el Desarrollo (GpRD), para lo cual analizaron la gestión tomando en cuenta el sistema de planificación, presupuesto, gestión financiera, gestión de programas y proyectos y la evaluación en 25 países, tomando una calificación en una escala de cero a cinco, donde el cinco se configura como óptima. La figura 1 ilustra los resultados hallados de la evaluación realizada, donde América Latina y el Caribe presenta un índice de desarrollo de 1.9, que nos describe que los países de la región se ubican al inicio de ejecución de la gestión orientada a resultados (BID, 2012).

\section{Figura 1}

Pilares de la Gestión para Resultados según su Nivel de Desarrollo

\begin{tabular}{|c|c|c|c|c|}
\hline \multirow[t]{2}{*}{ Pilar de la GpRD } & \multirow[t]{2}{*}{ Promedio } & \multicolumn{3}{|c|}{$\begin{array}{c}\text { Nivel de Desarrollo } \\
\text { GpRD }\end{array}$} \\
\hline & & Alto & Medio & Bajo \\
\hline Planificación para resultados & 2.3 & 3,5 & 2,3 & 1,6 \\
\hline Presupuesto por resultados & 1,4 & 3,1 & 1,3 & 0.5 \\
\hline Gestión financiera, auditoria y adquisiciones & 2,5 & 4,0 & 2,5 & 1,5 \\
\hline Gestión de programas y proyectos & 1,9 & 3,1 & 1,9 & 1,0 \\
\hline Monitoreo y evaluación & 1,6 & 3.8 & 1,3 & 0.7 \\
\hline Índice de GpRD & 1,9 & 3.5 & 1,9 & 1,1 \\
\hline
\end{tabular}

Nota: Resumen de la gestión institucional de paises de la región en los cinco pilares de gestión. Tomado de: "Buenas Prácticas de Gestión para Resultados en el Desarrollo en Latinoamérica y el Caribe" por Banco Interamericano de Desarrollo, 2012, p.11. Copyright 2012

Los países Latinoamericanos y el Caribe muestran un desarrollo desigual cuando se trata de la implementación en la Gestión para Resultado. Existen instituciones donde aún persiste la gestión burocrática tradicional, donde el presupuesto sigue siendo asignado en base a una lógica incrementalista y no en base a resultados. Lo contrario pasa con Chile que muestra índices (3.9) que supera al promedio regional, para lo cual ha impulsado la gestión en los programas. Seguido se encuentra, Colombia con un nivel medio para lograr implementar el Presupuesto por Resultados (García\&García, 2010). 
Asimismo, de los 25 países de América Latina en estudio, el Perú representa un país sobresaliente en el nivel intermedio para la Gestión para Resultados en el Desarrollo. El pilar que ha demostrado mayor desarrollo es en la gestión financiera, auditoría y adquisiciones $(3,1)$ superando el promedio regional $(2,5)$ y el pilar con bajo desarrollo es de monitoreo y evaluación $(1,2)$ puntaje por debajo del promedio regional $(1,6)$. Asimismo, Perú muestra mayor desarrollo después de Chile y Brasil en el componente de presupuesto por resultados (2.6), superando el promedio regional (1.4) (García\&García, 2010). La Nueva Gestión Pública (NGP), comprende un nuevo modelo de gestionar el Estado, incorporando una nueva forma de gerenciar las administraciones, reemplazando la forma tradicional entrega de servicios públicos, basados en el modelo burocrático de cumplimiento normativo por una gerencia publica basada en la eficacia y eficiencia (Larbi, 1999).

De la misma forma el presupuesto es apreciado como un instrumento de Gestión que concede a los entes Públicos la prestación de servicios con eficiencia y eficacia (Álvarez\&Álvarez, 2019). En ese orden de ideas el Presupuesto establecido para los gobiernos locales del Perú, se concentró en asignar recursos con un enfoque incrementalista y no sé basa en asignar recursos bajo el enfoque de resultados. Se tiene monto asignado a las municipalidades desde el año 2016 al 2020 ascendente a S/. 139, $615,137,453$ soles (MEF, 2020) y el impacto que generan no son evaluados. La evaluación constituye un apoyo para que los decisores tomen decisiones acertadas, en forma planificada de cada uno de los procesos (insumos, procesos, resultados) que nos permita generar recomendaciones para la mejora del desempeño de las entidades públicas (BID, 2014). Los gobiernos locales comprometidos con la etapa de ejecución de recursos financieros, no exponen parámetros de evaluación del presupuesto en base a logros, solo se enfocan en evaluar avances ligados a los ingresos y los gastos; sin embargo, no verifica la calidad con la que se presta los servicios.

Entre los años 2016 al 2020, la transferencia economía del Estado a la Municipalidad Provincial de Satipo se ha incrementado en un 183\%, es decir pasó de S/62,905,398.00 soles a S/82, 114,946,529.00 soles de presupuesto que administró la Municipalidad Provincial de Satipo, como se muestra en la tabla 1, logrando un promedio de ejecución del $74 \%$. 


\section{Tabla 1}

Ejecución presupuestal de la Municipalidad de Satipo (2016-2020)

\begin{tabular}{|c|c|c|c|c|c|}
\hline \multirow{2}{*}{ Año } & \multirow{2}{*}{ PIA } & \multirow{2}{*}{ PIM } & \multirow{2}{*}{ Certificación } & \multicolumn{2}{|c|}{ Ejecución } \\
\hline & & & & Devengado & $\%$ \\
\hline 2020 & $53,657,985$ & $114,946,529$ & $110,475,758$ & $70,002,266$ & $60.9 \%$ \\
\hline 2019 & $60,031,964$ & $82,776,267$ & $76,771,112$ & $61,434,705$ & $74.2 \%$ \\
\hline 2018 & $36,511,793$ & $105,403,740$ & $104,840,841$ & $91,186,918$ & $86.5 \%$ \\
\hline 2017 & $34,876,188$ & $106,356,114$ & $86,672,190$ & $58,709,113$ & $55.2 \%$ \\
\hline 2016 & $32,111,530$ & $62,905,398$ & $58,741,338$ & $53,198,271$ & $84.6 \%$ \\
\hline Total & $163,531,475$ & $357,441,519$ & $327,025,481$ & $264,529,007$ & $74.0 \%$ \\
\hline
\end{tabular}

Nota: Los datos son tomados de la consulta amigable del Ministerio de Economía y

Finanzas periodo 2016-2020. Fuente: Elaboración propia

La Municipalidad Provincial de Satipo, ha enfatizado en comprometer recursos del presupuesto institucional, de tal forma muchas veces el gasto no se ejecutó, razón por el cual no mejoraron la prestación de los servicios públicos. Es fundamental que aparte de orientarse en el avance de la ejecución financiara, también debe ir de la par con la calidad del gasto, a fin de estar de acorde con Armijo y Espada (2014) que señala "la calidad del gasto público abarca aquellos elementos que garantizan un uso eficaz y eficiente de los recursos públicos" (p.6). Las decisiones al inicio de la Gestión por Resultados en el gobierno local de Satipo aún son de naturaleza principiante se muestra una débil coordinación entre las unidades orgánicas y a nivel institucional entre Entidades con nivel de competencias transversales; con el peligro de incorporar actividades que no muestran relación y consistencia. La ejecución presupuestal de los últimos años no ha presentado variaciones profundas en sus formas de vida de los ciudadanos de Satipo, al contrario, los proyectos estuvieron orientados en infraestructura que no tuvieron efectos positivos en generar calidad de vida a la ciudadanía.

La capacidad de atender en forma satisfactoria las necesidades de la población y a la vez de una forma adecuada y en el tiempo oportuno, de acuerdo a los objetivos fijados, entonces sí efectivamente la gestión pública es de calidad (Andía, 2013). El censo de población, vivienda y comunidades indígenas desarrollado el año 2017, muestra que Satipo cuenta con 37,075 habitantes, con una pobreza de $18.7 \%$ y $2.01 \%$ de pobreza extrema al año 2013 (INEI 2018) y para el año 2019 presentan una tasa de desnutrición crónica de $14.6 \%$, desnutrición global de $4.6 \%$, desnutrición aguda $1.7 \%$ y riesgo de 
desnutrición crónica de $31.3 \%{ }^{1}$. Asimismo, los indicadores de anemia en niños menores de 12 meses bordean el $50.9 \%$ al primer semestre del 2019.

Asimismo, el MINEDU (2018) realizó la evaluación a los estudiantes a nivel censal, producto de ello especifica el $21.7 \%$ de estudiantes se encuentran previo al inicio de lectura, el $45.6 \%$ en inicio, $23.8 \%$ en proceso y $9 \%$ satisfactorio, del mismo modo en la prueba de matemáticas el $37.9 \%$ de estudiantes se encuentran en inicio, seguido del $15.0 \%$ en proceso y $10.8 \%$ satisfactorio. Otro aspecto importante es la insuficiente provisión de servicios básicos de agua y desagüe, el $65 \%$ de viviendas cuenta con agua potable y saneamiento vía red pública o pozo séptico, el $82 \%$ de las viviendas cuentan con electricidad, el 57\% de viviendas con gas y el 76\% con telefonía (INEI, 2018). Según la Municipalidad Provincial de Satipo (2017) el levantamiento de información a través de la Tecnología de Decisiones Informadas - TDI distrito de Satipo evidencio que el 100\% de viviendas presentan limitaciones en agua segura y desagüe, indicadores que contradice lo señalado por Delgado, Dimitrova, Guardiola y Shack (2016), que los gastos deben estar orientados a generar valor público para la población en consecuencia mejora la calidad de vida de la población.

\section{ESTRATEGIAS METODOLOGICAS O MATERIALES Y MÉTODOS}

\section{Tipo y diseño}

La investigación es de tipo aplicada en el cual se argumenta desde la posición de Naupas, Mejía, Novoa y Villagómez (2014), se "llaman aplicadas porque con base en la investigación básica, pura o fundamental, en las ciencias fácticas o formales, que hemos visto, se formulan problemas e hipótesis de trabajo para resolver los problemas de la vida productiva de la sociedad" (p. 61)

Asimismo, es de enfoque cuantitativo porque argumenta que es un proceso continuo, donde la información va ser recolectada para ser medidos y comprobar las hipótesis, para lo cual se desarrolla un análisis en base a la estadística con el propósito de establecer comportamientos y llegar a fundamentar teorías. También tiene un alcance correlacional, de diseño no experimental y transeccional o transversales, debido a que la investigación corresponde a un contexto real para después analizarlo en un tiempo señalado Sampieri, Fernández y Baptista (2014).

\section{Población y Muestra}

${ }^{1}$ Proporción de indicadores nutricionales en niños menores de 5 años que acceden a los establecimientos Ciencia Latina Revista Científica Multidisciplinar, Ciudad de México, México. 
En el estudio se contempló un tamaño de muestra de 28 servidores públicos involucrados en el ciclo de gastos como son presupuesto, administración y las unidades ejecutoras de la Municipalidad Provincial de Satipo con el propósito de alcanzar un análisis confiable.

\section{Instrumentos}

Se utilizó el instrumento del cuestionario con escalamiento Likert para medir las actitudes en las variables de gestión de presupuesto por resultados y calidad del gasto en la municipalidad provincial de Satipo. Asimismo, (Hernández\&Mendoza, 2019), señala que "cuestionario consiste en un conjunto de preguntas respecto a una o más variables a medirse" (p. 251). También se utilizó el instrumento de análisis documental, para lo cual se utilizó documentos de información, y fuentes de datos de la Entidades Públicas.

Para la confiabilidad del cuestionario se procedió a través del alfa de Cronbach, el cual dio un valor de 0,914 , lo que demuestra que los valores se ubican en el intervalo de 0,72 a 0,99, del alfa de Cronbach. Concluyendo que el instrumento que se utilizó tiene una excelente confiabilidad, demostrándose confiable para su aplicación.

\section{Procedimientos}

En el desarrollo del estudio se aplicó la estadística descriptiva y para conocer la relación entre las variables y la prueba de hipótesis se aplicó la estadística inferencial, a través del software estadístico SPSS. La técnica estadística para hallar la confiabilidad se demostró haciendo uso del Alpha de Cronbach y para comprobar la hipótesis se utilizó el coeficiente de Pearson. Además, se utilizó las técnicas estadísticas de medidas y correlación. En la investigación se usó el tipo de análisis cuantitativo y bivariado y asimismo para el desarrollo del estudio se aplicó los principios de confidencialidad y el consentimiento.

\section{RESULTADOS}

\section{Análisis correlacional}

De acuerdo a la tabla 2 queda demostrado que la variable de Gestión de Presupuesto por Resultado evidencia una correlación positiva modera con la variable de Calidad de Gasto. De tal manera que el coeficiente de Pearson, halló el valor de 0.693 y p-valor de 0.000 $(<0.01)$. 


\section{Tabla 2}

Prueba de correlación entre la Gestión de presupuesto por resultados y calidad del gasto

\begin{tabular}{llc}
\hline & & Calidad del gasto \\
\hline \multirow{2}{*}{$\begin{array}{l}\text { Gestión de presupuesto por } \\
\text { resultados }\end{array}$} & Coeficiente de Pearson &, $693^{* *}$ \\
& Sig. (bilateral) &, 000 \\
& $\mathrm{~N}$ & 28 \\
\hline \multirow{3}{*}{ Programa presupuestal } & Coeficiente de Pearson &, $647^{* *}$ \\
& Sig. (bilateral) &, 000 \\
& $\mathrm{~N}$ & 28 \\
\hline \multirow{3}{*}{ Seguimiento } & Coeficiente de Pearson &, $442^{* *}$ \\
& Sig. (bilateral) & .018 \\
& $\mathrm{~N}$ & 28 \\
\hline \multirow{3}{*}{ Evaluación } & Coeficiente de Pearson &, $729 * *$ \\
& Sig. (bilateral) &, 000 \\
& $\mathrm{~N}$ & 28 \\
\hline \multirow{3}{*}{ Evaluación } & Coeficiente de Pearson &, $729 * *$ \\
& Sig. (bilateral) &, 000 \\
& $\mathrm{~N}$ & 28 \\
\hline
\end{tabular}

Fuente: Elaboración propia

De igual forma demuestra que la dimensión programa presupuestal presenta una correlación positiva modera con la variable calidad del gasto, de tal manera que se obtuvo el estadístico de 0.647 y p-valor de $0.000(<0.01)$, por lo tanto, se concluye que efectivamente se presenta relación directa moderada del programa presupuestal con la calidad del gasto. También queda demostrado que la dimensión seguimiento tiene una correlación positiva moderada con la variable calidad del gasto, de tal manera que el nivel de correlación de Pearson, muestra el valor de 0.442 y p-valor de $0.018(<0.01)$.

Asimismo, teniendo en cuenta la tabla 2 la dimensión evaluación presenta una correlación positiva alta con la variable calidad del gasto en la municipalidad provincial de Satipo. De la misma manera el coeficiente de correlación de Pearson, halló que el estadístico es 0.729 y p-valor es $0.000(<0.01)$, por lo tanto, se confirma la existencia de correlación entre la evaluación y la variable de calidad del gasto. De la misma forma quedó demostrado que la dimensión incentivos tiene una relación positiva moderada con la variable calidad del gasto en la municipalidad de Satipo. De la misma manera el coeficiente de Pearson, halló que el estadístico es 0.540 y p-valor es $0.003(<0.01)$, por lo tanto, se confirma la existencia de correlación entre los incentivos a la gestión y la calidad del gasto. 


\section{Análisis descriptivo}

Conforme a la tabla 3, se aprecia de los 28 servidores civiles, el $43.9 \%$ (12) percibe según su experiencia que la gestión del presupuesto se orienta a resultados casi siempre, seguido del 34.4\% (10) en la valoración algunas veces, el 11.2\% (3) se presenta siempre, el 8,9\% se presenta muy pocas veces y finalmente el $1.5 \%$ no sé orientan a una Gestión de Presupuesto por resultados.

\section{Tabla 3}

Niveles de percepción de la variable gestión de presupuesto por resultados 2020

\begin{tabular}{lccc}
\hline \multicolumn{4}{l}{ Variable 1: Gestión de presupuesto por resultados (agrupado) } \\
\hline Valoración & Frecuencia & Porcentaje & $\begin{array}{c}\text { Porcentaje } \\
\text { acumulado }\end{array}$ \\
\hline Siempre & 3 & $11.2 \%$ & $11 \%$ \\
Casi siempre & 12 & $43.9 \%$ & $55 \%$ \\
Algunas veces & 10 & $34.4 \%$ & $90 \%$ \\
Muy pocas veces & 3 & $8.9 \%$ & $98 \%$ \\
Nunca & 0 & $1.5 \%$ & $100 \%$ \\
\hline \multicolumn{1}{c}{ Total } & $\mathbf{2 8}$ & $\mathbf{1 0 0 \%}$ & \\
\hline
\end{tabular}

Fuente: Elaboración propia

Se muestra la tabla 4, donde se señala que, de los 28 servidores civiles, el $46.4 \%$ percibe que los recursos públicos invertidos casi siempre son utilizados adecuadamente por la municipalidad, seguido del $35.7 \%$ que perciben algunas veces, también el $9.9 \%$ perciben muy pocas veces, $7.7 \%$ siempre y por último el $0.3 \%$ perciben que no son usados adecuadamente.

\section{Tabla 4}

Niveles de percepción de la variable de calidad del gasto 2020

\begin{tabular}{lccc}
\hline \multicolumn{1}{c}{ Variable 2: Calidad del Gasto (agrupado) } \\
\hline \multicolumn{1}{c}{ Valoración } & Frecuencia & Porcentaje & $\begin{array}{c}\text { Porcentaje } \\
\text { acumulado }\end{array}$ \\
\hline Siempre & 2 & $7.7 \%$ & $7.7 \%$ \\
Casi siempre & 13 & $46.4 \%$ & $54.1 \%$ \\
Algunas veces & 10 & $35.7 \%$ & $89.8 \%$ \\
Muy pocas veces & 3 & $9.9 \%$ & $99.7 \%$ \\
Nunca & 0 & $0.3 \%$ & $100.0 \%$ \\
\hline \multicolumn{4}{c}{ Total } \\
\multicolumn{4}{c}{$\mathbf{1 0 0 \%}$} \\
\hline
\end{tabular}

\section{Análisis de documentos y base de datos}

Según se aprecia en la tabla 5, los ingresos al 31 de diciembre del 2020 fueron de S/. 94,459,830.00 soles, sin considerar la fuente de Recursos Ordinarios, constituido por 
aquellos derivados de los programas de vaso de leche y de complementación alimentaria, con un indicador de eficacia de $99 \%$ en referencia al presupuesto modificado.

\section{Tabla 5}

Resumen de ingresos por rubro de financiamiento - indicador de eficacia 2020.

\begin{tabular}{|c|c|c|c|c|c|}
\hline \multirow[t]{2}{*}{ Rubro } & \multirow[t]{2}{*}{ PIA } & \multirow[t]{2}{*}{ PIM } & \multirow[t]{2}{*}{ Recaudado } & \multicolumn{2}{|c|}{$\begin{array}{c}\text { Indicador de } \\
\text { Eficacia }\end{array}$} \\
\hline & & & & PIA & PIM \\
\hline $\begin{array}{l}\text { 07: Fondo de compensación } \\
\text { municipal }\end{array}$ & $32,579,323$ & $32,227,160$ & $32,438,933$ & $100 \%$ & $101 \%$ \\
\hline 08: Impuestos municipales & $2,746,870$ & $2,185,206$ & $2,448,322$ & $89 \%$ & $112 \%$ \\
\hline $\begin{array}{l}\text { 09: Recursos directamente } \\
\text { recaudados }\end{array}$ & $4,268,651$ & $2,063,741$ & $2,231,107$ & $52 \%$ & $108 \%$ \\
\hline 13: Donaciones y transferencias & 0 & $2,331,907$ & $2,154,860$ & $0 \%$ & $92 \%$ \\
\hline $\begin{array}{l}\text { 18: Canon y sobre canon, regalías, } \\
\text { renta de aduanas y } \\
\text { participaciones }\end{array}$ & $3,077,619$ & $11,970,049$ & $11,195,703$ & $364 \%$ & $94 \%$ \\
\hline $\begin{array}{l}\text { 19: Recursos por operaciones } \\
\text { oficiales de crédito }\end{array}$ & 0 & $44,310,252$ & $43,990,905$ & $0 \%$ & $99 \%$ \\
\hline Total & $42,672,463$ & $95,088,315$ & $94,459,830$ & $221 \%$ & $99 \%$ \\
\hline
\end{tabular}

Fuente: Los datos fueron tomados del Portal de Transparencia Económica - MEF 2020. Elaboración: Propia, 2020

Para el ejercicio fiscal 2020 el monto del presupuesto pasó de S/53,657,985.00 soles a S/61,288,544.00 soles determinando un PIM de S/114,946,529.00 soles, mientras que la ejecución de los gastos ascendió a S/70,066,687.00 soles lográndose ejecutar el 61\% en referencia al PIM, resultado que pone en riesgo alto la eficiencia de ejecución del presupuesto. La ejecución presupuestaria fue $50 \%$ en el rubro 13 Donaciones y transferencia, el cual es poco alentador por la necesidad de los servicios básicos que se muestra en Satipo. En el rubro 00 Recursos Ordinarios respecto a lo programado en el PIM, el presupuesto se ha incrementado en $80.77 \%$ y que la ejecución de gastos fue de $65.4 \%$, con una posible reversión al tesoro público de 34.6\% (MEF, 2020).

Para el año 2020 la programación de los programas presupuestales ascendió de S/. 26,018,797.00 soles a la suma de S/.86,522,378.00 soles, obteniendo 52.8\% de eficacia del gasto, indicador que se encuentra por debajo del parámetro de 75\% (MEF toma como referencia para la ejecución de los programas presupuestales en forma anual). Los programas presupuestales que no alcanzaron la valla de $75 \%$ de ejecución presupuestal, fueron el programa de saneamiento urbano 9.6\%, el programa de saneamiento rural 
sistema de transporte de $42.5 \%$ y el programa de TBC-VIH/sida alcanzo el $20.5 \%$, ello demuestra que no se tuvo capacidad de gasto a pesar de las demandas sociales de los pobladores y a la vez representa alto riesgo expresado en la inadecuada ejecución anual (MEF, 2020).

Las inversiones realizadas durante el ejercicio fiscal 2020 ascienden al importe de S/.34,414,008.00 soles del PIM, da como resultado de 56.4\% de eficacia. En la cartera de inversiones se ha podido identificar 72 inversiones, que refleja la atomización de proyectos, en el cual se ha identificado que en Estudios de Preinversión programaron S/1,916,219.00 soles, pero solo ejecutaron 20.4\%. También hubo 23 proyectos con presupuestos efectivo que asciende a S/1,701,958.00, sin embargo, tuvieron 0.0\% de devengado, tal situación refleja la deficiencia en la programación de las inversiones, falta de seguimiento y personal poco preparada en el uso de los aplicativos de seguimiento de las inversiones y sobre todo falta de profesionalismo en el ingreso de información de los avances de ejecución de los proyectos (MEF, 2020).

La Municipalidad de Satipo logró cumplir las metas de los incentivos a la mejora de la Gestión de los Gobiernos Locales correspondientes al año 2020, ocupando el primer lugar a nivel de todo el País según el Ranking (Resolución Directoral N0022-2020-EF/50.01) y producto del cumplimiento recibió una transferencia por la suma de S/. 1,687,261.00 (Decreto Supremo N²09-2020-EF) y S/. 3,393,895.00 (Decreto Supremo N091-2021$\mathrm{EF}$ ), importe que permitió disponer de mayores recursos para la sostenibilidad de las actividades, sin embargo, fueron insostenibles y en caso de las inversiones solo se analiza el avance financiero, más no la calidad del gasto. Además, la Municipalidad de Satipo alcanzó cumplir con la meta de incentivo de Reconocimiento a la ejecución de las inversiones, alcanzado el $27.9 \%$ de avance en la ejecución de las inversiones (Resolución Directoral $\mathrm{N}^{\circ} 0006-2021-\mathrm{EF} / 50.0$ ).

El incremento de presupuesto de la Municipalidad de Satipo no se ve reflejado en la mejora d los servicios de saneamiento, educación, salud, residuos sólidos, seguridad, transporte entre otros. Asimismo, los servicios públicos brindados son limitados y no cuentan con estándares de calidad, lo que provoca resultados con indicadores insatisfactorios, como saneamiento que el $72 \%$ de la zona rural no tiene acceso al servicio de agua potable sean a través de la red o pileta pública y el $22 \%$ de la población no tiene acceso a alcantarillado u otros sistemas de disposición de excretas. También el 100\% de la población urbana no cuentan con servicio de drenaje pluvial y el $84 \%$ del volumen de aguas residuales no están tratadas (MEF, 2021), de lo descrito se desprende que las 
competencias exclusivas de las municipalidades no están siendo coberturados, a pesar que se ha analizado que los presupuestos no están siendo gastados al $100 \%$, del cual se detalla la poca atención de la municipalidad a solucionar los problemas de interés de la población

También en la provincia de Satipo el $80 \%$ de los puestos de Salud presentan inadecuada capacidad instalada y equipamiento y a la vez pertenecen al primer nivel de atención, el $25 \%$ de viviendas de la zona urbana de Satipo no acceden a servicios de energía eléctrica, seguido del 18\% de la zona rural con ausencia de servicio eléctrico, el 98\% de las escuelas presentan capacidad instalada inadecuada, el 94\% de los colegios presentan inadecuada capacidad instalada, el 90\% de instituciones de nivel inicial presentan infraestructuras en condiciones inadecuadas (MEF, 2021). También en referencia al transporte, el 86\% de la población de la zona urbana se encuentran sin acceso a pistas y veradas adecuadas, el $70 \%$ de los caminos de herradura no fueron intervenidos, el $68 \%$ de la red vial vecinal se encuentra no pavimentada y el 50\% de red vial vecinal sin pavimentar (MEF, 2021).

Por último, se tiene la función ambiente, donde el $100 \%$ de los gobiernos locales y regionales presentan capacidades operativas inadecuadas en la prestación de los servicios de ordenamiento territorial, también el $83 \%$ de los espacios verdes en las zonas urbanas no son atendidos y el $62 \%$ no cuentan con un servicio adecuado de limpieza pública, brechas que reflejan lo poca capacidad del gobierno local de asumir competencias exclusivas para solucionar dificultades en la administración de residuos sólidos (MEF, 2021). 


\section{DISCUSIÓN}

La investigación encontró que la gestión de presupuesto por resultados y calidad de gasto en la Municipalidad Provincial de Satipo, mantienen una relación positiva moderada $\left(0.693^{* *}\right)$ y significativa $(\mathrm{p}=0.000<0.01)$, similar a la de García $(2020)$ que también muestra una relación positiva moderada del presupuesto por resultado y calidad del gasto público en la municipalidad de Huarmaca, respaldándose con un Rho Spearman de 0.642 y su nivel de significancia bilateral $\mathrm{p}=0.000<0.01$ ). De la misma manera coincide con Vargas y Zavaleta (2020), quien afirma que la gestión de presupuesto por resultados se relaciona en forma significativa con la variable calidad del gasto, con un modelo estadístico $[$ Prob $($ F Statistic $)=0.0000$ menor a 0.05 de nivel de significancia, igualmente se comprueba con el coeficiente de 0.69 de correlación. También se encontró que el 43.9\% de los funcionarios perciben que casi siembre se manifiesta la Gestión del Presupuesto por Resultados en la municipalidad de Satipo, seguido del $46.4 \%$ perciben que casi siembre los recursos se invierten con eficiencia. Esto a su vez corrobora la teoría de Arbaiza, Álvarez, Hernández, Sarria y Villalba (2017), donde afirma que la calidad del gasto viene hacer cuando se usa en forma eficaz y eficiente los recursos del Estado para lograr objetivos que contribuyan al crecimiento de la economía.

El presupuesto administrado por la municipalidad de Satipo se distribuye un $60 \%$ en gastos corrientes y un $40 \%$ en gastos de inversión y año tras años la asignación del presupuesto es en base al enfoque incremental del presupuesto tradicional así como lo señala García y García (2010) los países de Latinoamérica y el caribe redistribuyen el presupuesto de modo incrementalista, que consisten en el incremento del presupuesto año tras año sin una lógica de logro de resultados en transporte, seguridad, educación, salud y saneamiento, resultado que contrasta con la teoría de Shack y Rivera (2017) donde señala que la asignación de los recursos debe estar vinculado al logro de resultados expresado a su vez que el presupuesto se debe clasificar de acuerdo a los programas y a la vez deben evaluarse la efectividad del gasto público como también de los incentivos. La relación entre el programa presupuestal y la calidad de gasto, reveló una correlación positiva moderada y significativa; resultado que coincide con Gutiérrez (2017), donde señala que los programas presupuestales con la calidad y eficiencia de la gestión, presenta un valor de correlación de Spearman igual a 0.847. Asimismo, el $42.9 \%$ de los funcionarios perciben que casi siempre los programas presupuestales mejoran las condiciones de bienestar, resultado que corrobora Andía (2013) donde afirma que los programas presupuestales es una forma de programación que se enfoca en la prestación 
de bienes y servicios con el propósito de obtener resultados en beneficio de la población objetivo. De igual forma el MEF (2016) señala que los programas presupuestales son estrategias que se enfocan en vincular los recursos públicos a resultados que sean medibles para beneficiar a la población

La ejecución financieramente se da al 100\% para cumplir metas porque se entiende que cumpliendo metas se demuestra eficiencia en el gasto, el cual contrasta la teórica de García y García (2015), donde señala que la reasignación de recursos debe ser en base a resultados para lograr efectividad y eficiencia, más no la lógica incremental. Además, el $75.3 \%$ del presupuesto se asignó a programas presupuestales que permita obtener resultados, el mismo coincide con Andía (2013), que indica que una buena práctica de programación de los recursos de la Entidad debe obedecer en base a programas presupuestales que integrados y articulados beneficia a la población objetivo. En la Municipalidad Provincial de Satipo (2021) por motivos del Estado- de Emergencia por la COVID 19 hubo recorte presupuestal de un 35\% que impidió que las unidades orgánicas en muchos casos no lograron los Objetivos Estratégicos Institucionales del Plan Estratégicos Institucional.

Referente a la relación del seguimiento y la calidad de gasto, se confirma la relación positiva baja y significativa, resultado que se asemejan con Gutiérrez (2017), donde señala que existe una relación directa y significativa entre la herramienta de seguimiento, evaluación y calidad de gestión de los recursos públicos con un coeficiente de correlación de 0.784 y el p-valor de $0.000(<0.01)$. También el $19.6 \%$ de los funcionarios perciben que siembre toman acciones correctivas para evitar corregir deficiencias y a la vez cuentan con plan de seguimiento para el desarrollo de actividades e inversiones. Ello concuerda con la teoría de Álvarez (2011) donde afirma que el seguimiento debe ser de carácter permanente para el avance de los gastos y la parte física de actividades e inversiones con el propósito de tomar acciones correctivas oportunas cuando se presenta deficiencias en el ciclo del gasto. De acuerdo a la revisión documental el Gobierno Local no cuentan con instrumentos para realizar seguimiento al avance físico y financiero de las inversiones, resultado que no sé alinea con el Ministerio de Economía y Finanzas al señalar que la fase de seguimiento es permanente con el propósito de contrastar la obtención de resultados.

La relación de la evaluación y la calidad de gasto, es positiva alta y significativa que guardan relación con los hallazgos de Castillo (2016), donde afirma que los indicadores 
de 0.000 (menor del 0.01) en forma positiva muy fuerte de 0.919. De la revisión documental la evaluación de Plan Estratégico Institucional 2020 señala que el 89\% de las Gerencias responsables de los Objetivos Estratégicos del Plan Estratégicos Institucional presentan una débil cultura en la evaluación de las intervenciones, resultado que no se alinea con la teoría de Shack y Rivera (2017), donde revela que la evaluación es un asunto ordenado de diseño, desarrollo y resultados de la intervención pública para determinar la eficacia.

Por último, en referencia a la relación del incentivo y la calidad del gasto, presentan correlación positiva moderada y a su vez significativa, el mismo que contrasta con Jacobo (1018), en el cual afirma que el cumplimiento de metas y los incentivos recibidos tienen relación alta positiva, siendo el coeficiente de correlación múltiple de 0.749 y el p-valor de $0.000(<0.01)$. En los resultados descriptivos el $50 \%$ y $7.1 \%$ los funcionarios perciben que siempre y casi siembre respectivamente consideran que los incentivos otorgados a la Municipalidad contribuyen a alcanzar de manera significativa los objetivos institucionales. Esto a su vez corrobora la teoría de García y García (2015), donde afirma que los incentivos estimulan el logro de propósito de los individuos, equipos de trabajo e instituciones. 


\section{CONCLUSIONES}

Se determina que la gestión de presupuesto por resultado y la calidad del gasto de la municipalidad de Satipo se relacionan, con el coeficiente de Pearson de 0,693, porque implementaron estrategias de administración que permita vincular los presupuestos a productos y a resultados que sean medibles para la mejora de la calidad del de gasto. No obstante, la ejecución presupuestal fue de $61.0 \%$, indicador de eficacia que no se plasma en la mejoría de vida de los habitantes de Satipo, a causa de la existencia de brechas de infraestructura y servicios públicos.

Los programas presupuestales se relacionan con la calidad del gasto, con una correlación de Pearson de 0,647, comprobándose de esta manera que el $72.7 \%$ de los recursos presupuestados se orientó a programas presupuestales, sin embargo, la asignación del presupuesto no necesariamente se enfocó al cierre de brechas.

También el seguimiento y la variable calidad del gasto se consiguió un coeficiente de 0,442, que denota la existencia de una correlación positiva moderada, debido a que la municipalidad tiene como política institucional el seguimiento de las intervenciones con el propósito de que las intervenciones muestran mejoría en la calidad del gasto, sin embargo se muestra deficiencia en la programación financiera y física de las inversiones debido al desconocimiento de herramientas de programación, el cual ha ocasionado en reiteradas oportunidades el incumplimiento de los acuerdos adoptados en el equipo de seguimiento de las Inversiones.

Además, se determinó la relación entre la dimensión de evaluación y la variable de calidad del gasto con un valor de Pearson de 0,729, el cual señala una correlación positiva alta, esto evidencia que la municipalidad al implementar la evaluación de las intervenciones con indicadores medibles entonces ayuda a que la calidad del gasto se mejore. Sin embargo, la evaluación del Plan Estratégico Institucional de la Entidad muestra que los funcionarios no tienen una cultura de evaluación de las actividades.

Finalmente, los incentivos presentan relación con la calidad del gasto con un valor de Pearson de 0,540, toda vez que la municipalidad implemento estrategias de incentivos a la ejecución de actividades, programas y/o inversiones para que la calidad del gasto se mejore, expresado en el logro de metas fijadas por los incentivos Municipales en el ejercicio fiscal 2020, pero que no necesariamente mejoraron la calidad del gasto. 


\section{REFERENCIAS BIBLIOGRÁFICAS}

Álvarez, A., \& Álvarez, O. (2019). Presupuesto Público Comentado 2019: Presupuesto por Resultados y Presupuesto Participativo. Pacificos Editores S.A.C.

Álvarez, J. (2011). Gestión por Resultados e Indicadores de Medición. Pacifico Editores S.A.C.

Andía, W. (2013). Manual de Gestión Pública: Pautas para la Aplicación de los Sistemas Administrativos (Tercera ed.). El Saber.

Arbaiza, L., Álvarez, P., Hernández, C., Sarria, S., \& Villalba, E. (2017). Monitoreo del Programa Presupuestal Salud Materno Neonatal para el sector público. Universidad ESAN.

Armijo, M., \& Espada, M. (2014). Calidad del gasto público y reformas institucionales en Ámerica Latina. Naciones Unidas.

Banco Interamericano de Desarrollo. (2012). Buenas prácticas de Gestión para el Desarrollo en Latinoamerica y el Caribe. Banco Interamericano de Desarrollo.

Banco Interamericano de Desarrollo. (2014). Presupuestos para el desarrollo en América Latina. New York: A\&S Information Specialists, LLC.

Castillo, R. (2016). Relación de la Gestión Presupuestal con el gasto municipal del distrito de Morales - 2015 [Tesis de maestria, Universidad César Vallejo]. Repositorio Institucional. Obtenido de https://repositorio.ucv.edu.pe/bitstream/handle/20.500.12692/5398/castillo_sr.pd f? sequence $=1$ \&isAllowed $=\mathrm{y}$

Delgado, S., Dimitrova, D., Guardiola, U., \& Schack, N. (2016). Fiscalización y Calidad del Gasto Público en América Latina en América Latina y El Caribe:. F\&G Editores.

Garcia, k. (2020). Presupuesto por resultados y su relación con la calidad del gasto público en la municipalidad Distrital de Huarmaca, Piura, 2018 [Tesis de Maestria, Universidad César Vallejo]. Repositorio Institucional. Obtenido de https://repositorio.ucv.edu.pe/bitstream/handle/20.500.12692/45834/Garcia_TK L-SD.pdf?sequence=1\&isAllowed=y

Garcia, M., \& Garcia, R. (2015). Aspectos conceptuales y metodologicos. En J. Kaufmann, M. Sanginés, \& M. Garcia, Construyendo gobiernos efectivos: Logros y retos de la gestión pública para resultados en América Latina y el Caribe (2da ed., págs. 1-24). Sarah Scshineller (A\&S Information Specialists, LLC). 
Garcia, R., \& Garcia, M. (2010). La Gestión para Resultados en el Desarrollo: Avances y desafios en América Latina y el Caribe. Banco Interamericano de Desarrollo.

Gutierrez, J. (2017). Programa presupuestal PpR, calidad y eficiencia de gestión de los recursos públicos, de la Dirección de Salud Apurímac II [Tesis de Doctorado, Universidad César Vallejo]. Repositorio Institucional. Obtenido de https://repositorio.ucv.edu.pe/bitstream/handle/20.500.12692/4456/Gutierrez_M JA.pdf?sequence $=1 \&$ isAllowed $=\mathrm{y}$

Hérnandez, R., \& Mendoza, C. (2019). Metodología de la investigación: Las rutas cuantitativa, cualitativa y mixta. McGraw-Hill Internamericana Editores, S.A. de C.V.

Instituto Nacional de Estadística e Informática. (octubre de 2018). publicaciones_digitales Censos Nacionales 2017: XII de Población, VII de Vivienda y III de Comunidades Indígenas. Obtenido de Instituto Nacional de Estadística $\mathrm{e}$ Informática: https://www.inei.gob.pe/media/MenuRecursivo/publicaciones_digitales/Est/Lib1 $576 /$

Jacobo, F. (2018). Programa de incentivos y su impacto en la mejora de la gestión de la municipalidad distrital de Usquil, periodo 2014-2015 [Tesis de Maestria, Universidad Cesar Vallejo]. Repositorio Institucional. Obtenido de https://repositorio.ucv.edu.pe/bitstream/handle/20.500.12692/11805/jacobo_nf.p df? sequence $=1 \&$ is Allowed $=\mathrm{y}$

Larbi, G. (1999). The New Public Management Approach and Crisis States. Instituto de Investigaciones de las Naciones Unidas para el Desarrollo Social (UNRISD).

Ministerio de Economia y Finanzas. (2016). Programación multianual y formulación anual del presupuesto para programas presupuestales con articulación territorial (Primera edición ed.). Lima, Perú: Vértice Consultores. Obtenido de https://www.mef.gob.pe/contenidos/presu_publ/ppr/prog_presupuestal/guia_pro g_multianual.pdf

Ministerio de Economia y Finanzas. (11 de noviembre de 2020). Seguimiento de la Ejecución Presupuestal. Obtenido de Portal de Transparencia Económica: https://apps5.mineco.gob.pe/transparencia/mensual/

Ministerio de Economia y Finanzas. (2020, 19 de julio). Resolución Directoral $\mathrm{N}^{\circ} 0022$ 2020-EF/50.01. Aprueba los resultados de la evaluación del cumplimiento y el 
Incentivos a la Mejora de la Gestión Municipal establecidas al 15 de junio del año 2020. Diario Oficial El Peruano. Obtenido de https://www.mef.gob.pe/es/porinstrumento/resolucion-directoral/22923-resolucion-directoral-n-0022-2020-ef50-01/file

Ministerio de Economia y Finanzas. (2020, 31 de julio). Decreto Supremo N²09-2020EF. Autorizan transferencia de Partidas en el Presupuesto del Sector Público para el Año Fiscal 2020 a favor de Gobiernos Locales en el marco del Programa de Incentivos a la Mejora de la Gestión Municipal. Diario Oficial El Peruano. Obtenido de https://www.mef.gob.pe/es/por-instrumento/decretosupremo/23061-decreto-supremo-n-209-2020-ef/file

Ministerio de Economia y Finanzas. (2021). Reporte Departamental y Distrital de Indicadores de Brechas. Obtenido de 20 junio del 2021 de https://ofi5.mef.gob.pe/brechas

Ministerio de Economia y Finanzas. (2021, 19 de febrero). Resolución Directoral $\mathrm{N}^{\circ} 0006-2021-\mathrm{EF} / 50.01$. Aprueba los resultados de la evaluación del cumplimiento de las metas del Reconocimiento a la Ejecución del año 2020. Diario Oficial El Peruano. Obtenido de https://www.mef.gob.pe/es/porinstrumento/resolucion-directoral/25022-resolucion-directoral-n-0006-2021-ef50-01/file

Ministerio de Economia y Finanzas. (2021, 29 de abril). Decreto Supremo Nº91-2021EF. Autorizan transferencia de partidas en el Presupuesto del Sector Público para el año Fiscal 2021 hasta por la suma de S/ 600000 000,00 a favor de diversos Gobiernos Locales por el cumplimiento de metas establecidas al 31 del año 2020. Diario Oficial el Peruano. Obtenido de https://cdn.www.gob.pe/uploads/document/file/1860082/DS091_2021EF.pdf.pd $\mathrm{f}$

Ministerio de Educación. (2018). Resultados de la Evaluación Censal de Estudiantes $20184^{\circ}$ grado de primaria $/ 2^{\circ}$ grado de secundaria. Obtenido de 14 de junio del 2021 de http://umc.minedu.gob.pe/wp-content/uploads/2019/06/DREJun\%C3\%ADn-2016-Marzo-2019.pdf

Municipalidad Provincial de Satipo. (2017). Tecnologia de Decisiones Informadas - TDI distrito de Satipo. Municipalidad Provincial de Satipo. Recuperado el 14 de abril de 2020 
Municipalidad Provincial de Satipo. (30 de marzo de 2021). Informe de evaluación de resultados del Plan Estratégico Institucional PEI 2018-2023 del año 2020. Obtenido de Portal de Transparencia Estándar: https://drive.google.com/file/d/1DRPGeiGYitwEao6Akv8wQ10UQrCcXtT/view

Ñaupas, H., Mejía, E., Novoa, E., \& Villagómez, A. (2014). Metodologia de la Investigación cuantitativa-cualitativa y redacción de tesis (Tercera ed.). Xpress Estudio Gráfico y Digital S.A.

Sampieri, R., Fernández, C., \& Baptista, P. (2014). Metodologia de la Investigación (Sexta ed.). McGraw-Hill/Interamericana Editores, S.A. de C.V.

Shach, N., \& Rivera, R. (2017). Seis años de la Gestión Pública para Resultados en el Perú. Universidad Continental Fondo Iditorial.

Vargas, J., \& Zavala, W. (2020). La Gestión de Presupuesto por Resultados y la Calidad del Gasto en Gobiernos Locales. Visión de Futuro, 24(2), 37-59. doi:https://doi.org/10.36995/j.visiondefuturo.2020.24.02.002.es 